\title{
Convergent and divergent phenomena of tidal current constituents in the Yellow Sea and the Bohai Sea
}

\author{
Lei Huang ${ }^{1, a}$, A.Wall ${ }^{2, b}$ \\ ${ }^{1}$ Department of Marine, Qingdao Ocean Shipping Mariners College, Qingdao, 266071, China \\ ${ }^{2}$ School of Engineering, Technology and Maritime Operations, Liverpool John Moores University, \\ Liverpool, L3 3AF, UK \\ a huanglei@coscoqmc.com.cn, ${ }^{b}$ A.D.Wall@ljmu.ac.uk
}

Keywords: the Yellow Sea; the Bohai Sea; tidal constituents; tidal currents; properties of tide and tidal current

Abstract. In the Yellow Sea and the Bohai Sea, the five layers tidal currents fields of $\mathrm{M}_{2}, \mathrm{~S}_{2}, \mathrm{~K}_{1}$ and $\mathrm{O}_{1}$ constituents were computed hourly in a period based on the three-dimensional model established by Jan O. Backhaus. The computation results indicate that three convergent and divergent regions of tidal currents exist in the junctures of four semidiurnal tide wave systems, and one exists in the juncture of two diurnal tide wave systems. The convergence and divergence of tidal current constituents are the probable cause of dissimilarity between the tide type and tidal current type.

\section{Introduction}

The Yellow Sea and the Bohai Sea are shelf seas along coast of China, where the energies of tide and tidal current are large due to the influx of Pacific tide wave. The tide and tidal current are important processes which affect the distributions of temperature, salinity and the feature of circulation. Understanding the characteristics of the tide and tidal current in these sea areas are important for the safety of port and harbor construction, mudflat aquaculture, marine activities, etc.

In the past decades, there are many numerical studies ${ }^{[1-5]}$ on tide and tidal current in the Yellow Sea and the Bohai Sea. From these studies, many characteristics of the tide and tidal current in these sea areas have been explained, but the characteristic and cause of dissimilarity between the tide type and tidal current type have had no effective interpretation.

In this paper, based on the three-dimensional model established by Backhaus ${ }^{[6,7]}$, the five layers tidal currents fields of $\mathrm{M}_{2}, \mathrm{~S}_{2}, \mathrm{~K}_{1}$ and $\mathrm{O}_{1}$ constituents were computed hourly in a period in the Yellow Sea and the Bohai Sea. Meanwhile, the harmonic analyses for the measured data from tens of stations were performed. The result of numerical model agrees well with the harmonic analysis. Therefore, convergent and divergent phenomena of tidal current constituents and causes of dissimilarity between the tide type and tidal current type in these sea areas have been explained.

\section{Calculation method}

Vertical arrangement of grid-points in an $\mathrm{x}-\mathrm{z}$ section and horizontal distribution of grid-points are illustrated in Fig. 1 and Fig. 2.

In the computational sea area, the depth of water is separated into $L$ levels and the thickness of each level is $h_{1}+\zeta, h_{2} \mathrm{~L} h_{L}$ respectively.

In a Cartesian system with

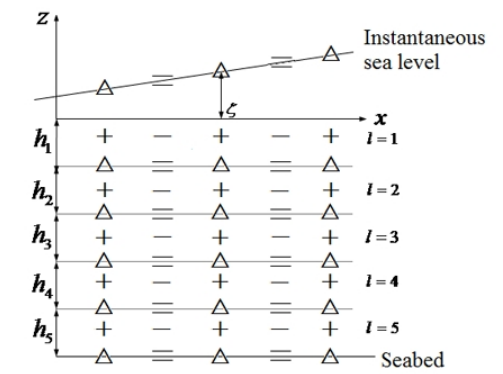

Fig. 1. Vertical arrangement of grid-points in an $\mathrm{x}-\mathrm{z}$ section

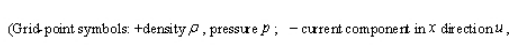

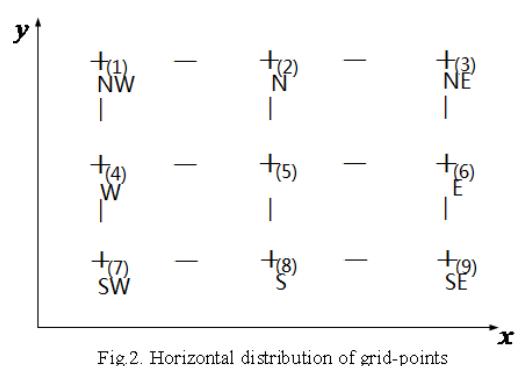

(Grid. point symbols: +surface elevation 6 , pressure

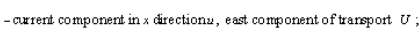

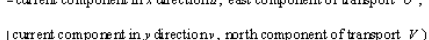
horizontal coordinates $x, y$ and vertical coordinate $z$, vertically upward from the mean sea level, 
the equations of motion and the continuity equation are vertically integrated over a depth range $h_{l}$, the follow equations are obtained:

$$
\begin{aligned}
& \frac{\partial U_{l}}{\partial t}-f V_{l}+\left(\frac{h}{\rho}\right)_{l}\left(g \rho_{1} \frac{\partial \zeta}{\partial x}+\frac{\partial I}{\partial x}\right)=X+\Delta \tau_{l}^{x} \\
& \frac{\partial V_{l}}{\partial t}+f U_{l}+\left(\frac{h}{\rho}\right)_{l}\left(g \rho_{1} \frac{\partial \zeta}{\partial x}+\frac{\partial I}{\partial y}\right)=Y+\Delta \tau_{l}{ }_{l} \\
& \frac{\partial U_{l}}{\partial x}+\frac{\partial V_{l}}{\partial y}+\Delta w_{l}=0
\end{aligned}
$$

where

$$
l=1,23, \ldots, L
$$

$$
\begin{array}{lll}
X_{l}=-\frac{\partial(u U)_{l}}{\partial x}-\frac{\partial(u V)_{l}}{\partial y}-(u w)_{l-\frac{1}{2}}+(u w)_{l+\frac{1}{2}}+A_{H}\left(\frac{\partial^{2} U}{\partial x^{2}}+\frac{\partial^{2} U}{\partial y^{2}}\right)_{l}, & Y_{l}=-\frac{\partial(v U)_{l}}{\partial x}-\frac{\partial(v V)_{l}}{\partial y}-(v w)_{l-\frac{1}{2}}+(v w)_{l+\frac{1}{2}}+A_{H}\left(\frac{\partial^{2} V}{\partial x^{2}}+\frac{\partial^{2} V}{\partial y^{2}}\right)_{l}, \\
\tau_{l}^{x}=A_{V} \frac{\partial u}{\partial z}=\frac{A_{V}}{\left(h_{l-1}+h_{l}\right) / 2}\left[\left(\frac{U}{h}\right)_{l-1}-\left(\frac{U}{h}\right)_{l}\right], & \tau_{l}^{y}=A_{V} \frac{\partial v}{\partial z}=\frac{A_{V}}{\left(h_{l-1}+h_{l}\right) / 2}\left[\left(\frac{V}{h}\right)_{l-1}-\left(\frac{V}{h}\right)_{l}\right] .
\end{array}
$$

where $u 、 v$ and $w$ are the current components in $x, y$ and $z$ directions respectively; $U_{l}=(u h)_{l} ; V_{l}=(v h)_{l} ; \zeta$ is the elevation; $f$ is the Coriolis parameter; $A_{V}$ and $A_{H}$ denote the vertical and horizontal eddy viscosity parameters; $\rho$ denotes the density, $I$ denotes the baroclinic parameter, and $\tau$ denotes the shear stress.

The explicit difference scheme is employed in handling $X, Y$ and $I$. Then, Eq. (1) and Eq. (2) can be written as

$$
\begin{aligned}
& U_{l}^{n+1}=\alpha U_{l}^{n}+\beta V_{l}^{n}-\left(\frac{h}{\rho}\right) \frac{1}{f}\left[\rho_{1} g\left(\beta \frac{\partial \zeta}{\partial x}+\gamma \frac{\partial \zeta}{\partial y}\right)^{n+\frac{1}{2}}+\left(\beta \frac{\partial I}{\partial x}+\gamma \frac{\partial I}{\partial y}\right)_{l}^{n}\right]+\Delta t X_{l}^{n}+\Delta\left(\tau_{l}^{x}-\tau_{l+1}^{x}\right)^{n+\frac{1}{2}} \\
& V_{l}^{n+1}=\alpha V_{l}^{n}-\beta U_{l}^{n}-\left(\frac{h}{\rho}\right)_{l} \frac{1}{f}\left[\rho_{1} g\left(-\gamma \frac{\partial \zeta}{\partial x}+\beta \frac{\partial \zeta}{\partial y}\right)^{n+\frac{1}{2}}+\left(-\gamma \frac{\partial I}{\partial x}+\beta \frac{\partial I}{\partial y}\right)_{l}^{n}\right]+\Delta t Y_{l}^{n}+\Delta\left(\tau_{l}^{y}-\tau_{l+1}^{y}\right)^{n+\frac{1}{2}}
\end{aligned}
$$

where $\alpha=\cos (f \Delta t) ; \beta=\sin (f \Delta t) ; \gamma=1-\cos (f \Delta t)$, and the exponential $n+\frac{1}{2}$ denotes the auxiliary time step in the middle of the $n$th and $(n+1)$ th time steps.

On the basis of Eq. (3), the equation for predicting the elevation can be obtained as follows:

$$
\zeta^{n+1}-\frac{\Delta t g}{4 f} \frac{\partial}{\partial x}\left[\overline{\left(\frac{h}{\rho}\right)} p_{1}\left(\beta \frac{\partial \zeta^{n+1}}{\partial x}+\gamma \frac{\partial \zeta^{n+1}}{\partial y}\right)\right]-\frac{\Delta t g}{4 f} \frac{\partial}{\partial y}\left[\overline{\left(\frac{h}{\rho}\right)} p_{1}\left(-\gamma \frac{\partial \zeta^{n+1}}{\partial x}+\beta \frac{\partial \zeta^{n+1}}{\partial y}\right)\right]=\frac{\Delta t}{2}\left[A_{3}-\left(\frac{\partial A_{1}}{\partial x}+\frac{\partial A_{2}}{\partial y}\right)\right]
$$

where $A_{1}, A_{2}$ and $A_{3}$ are computed based on the known variables $U, V$ and $\zeta$ etc. which are obtained at the $n$th time step.

By substituting Eq. (3) into Eq. (6), the linear system of equations, which is the final version of horizontal implicit system, is obtained:

$$
\zeta_{i, j}^{K+1}=(1-\omega) \zeta_{i, j}^{K}+\frac{\omega}{1+C_{5}}\left(B_{i, j}+C_{i, j}+C_{1} \zeta_{i-1, j+1}^{K+1}+C_{2} \zeta_{i, j+1}^{K+1}+C_{3} \zeta_{i+1, j+1}^{K+1}+C_{4} \zeta_{i-1, j}^{K+1}+C_{7} \zeta_{i-1, j-1}^{K}+C_{8} \zeta_{i, j-1}^{K}+C_{9} \zeta_{i+1, j-1}^{K}\right)
$$

where $\omega$ is the relaxation parameter; $B$ and $C$ are computed based on the known variables $U$, $V$ and $\zeta$ of the $n$th time step; $C_{1}, C_{2}, \mathrm{~L}, C_{9}$ are based on the known variables $\Delta t, \Delta x, \Delta y$ and the depth of each point. Fig.2 shows the positions from 1 to 9 .

By using the method of successive over-relaxation (SOR), the solution of Eq. (7) is obtained. By substituting the elevation of the $(n+1)$ th time step into Eq. (4) and Eq. (5), the vertical implicit system is obtained:

$$
\begin{aligned}
& -a_{l} U_{l-1}^{n+1}+b_{l} U_{l}^{n+1}-c_{l} U_{l+1}^{n+1}=d_{x, l} \\
& -a_{l} V_{l-1}^{n+1}+b_{l} V_{l}^{n+1}-c_{l} V_{l+1}^{n+1}=d_{y, l}
\end{aligned}
$$

System (8) and (9) are tri-diagonal, where the elements $a, b, c$ and $d$ are known.

By using the speedup method in solving Eq. (8) and Eq. (9), the horizontal currents of the $(n+1)$ th time step are obtained. By substituting the horizontal currents of each time step into the continuity equation: 


$$
w_{l}=-\sum_{K=l}^{L}\left(\frac{\Delta \mathrm{U}}{\partial x}+\frac{\Delta \mathrm{V}}{\partial y}\right)_{K},
$$

$$
l=12 \cdots \mathrm{L}
$$

The vertical currents of each time step are also obtained. By using the time-average over a tidal period for the horizontal and vertical currents, the tide-induced horizontal and vertical residual currents are finally obtained.

In this paper, the model domain includes the Bohai Sea and the Yellow Sea north of $33^{\circ} \mathrm{N}$, and the horizontal grid spacing is as follows: $\Delta y=5 \times 1.852 \times 10^{5} \mathrm{~cm}, \Delta x=\Delta y \cos \phi$.

The water depth is separated 5 levels in the vertical direction, i.e., $\mathrm{L}=5$. The thickness is 5 $\mathrm{m}$ for level 1, $7 \mathrm{~m}$ for level 2, $8 \mathrm{~m}$ for level 3, $10 \mathrm{~m}$ for level 4, and the remaining depth for level 5 .

In the given equations, the horizontal eddy viscosity parameter $A_{H}$ is $10^{7} \mathrm{~cm}^{2} / \mathrm{s}$, the vertical eddy viscosity parameter $A_{V}$ is $8 \mathrm{~cm}^{2} / \mathrm{s}$, the bottom frictional factor $r$ is $10^{-4}, \rho$ is constant, $I$ is zero when the barotropic model is used, $\tau_{1}$ is zero when the effect of wind is ignored.

At the solid boundary, the current speed in the normal direction is zero. At the open boundary, the forced water level is given as follow:

$$
\zeta_{i, j}=H_{i, j} \cos \left(\sigma n-g_{i, j}\right)
$$

The time step is as follows: $\Delta t=T / N$,where $T$ is the cycle of tide, $N$ is the number of time steps, $N_{M_{2}}=360, N_{S_{2}}=360, N_{K_{1}}=720, N_{O_{1}}=720$.

Harmonic constants of tidal constituents are computed based on the elevation $\zeta$ of tidal constituents at grid-points. Harmonic constants of tidal current constituents are computed based on the current components $u$ and $v$. Then the properties of tide and tidal current at grid-points are confirmed.

\section{Results}

\section{Convergent and divergent phenomena of the tidal current constituents in the Yellow Sea and the Bohai Sea}

The tidal currents of $\mathrm{M}_{2}, \mathrm{~S}_{2}, \mathrm{~K}_{1}$, and $\mathrm{O}_{1}$ constituents in the Bohai Sea and the Yellow Sea were computed hourly in a period,using the three-dimensional nonlinear numerical model presented by Jan.O. Backhaus. Fig.3 and Fig.4 show the numerical calculated distributions of tidal current of $\mathrm{M}_{2}$ constituent at $00 \mathrm{~h}$ and $06 \mathrm{~h}$ in surface layer. Meanwhile, the harmonic analyses for the measured data from tens of stations are performed. Measured distributions are illustrated in Fig.5 and Fig.6. The result of numerical model agrees well with the harmonic analysis.
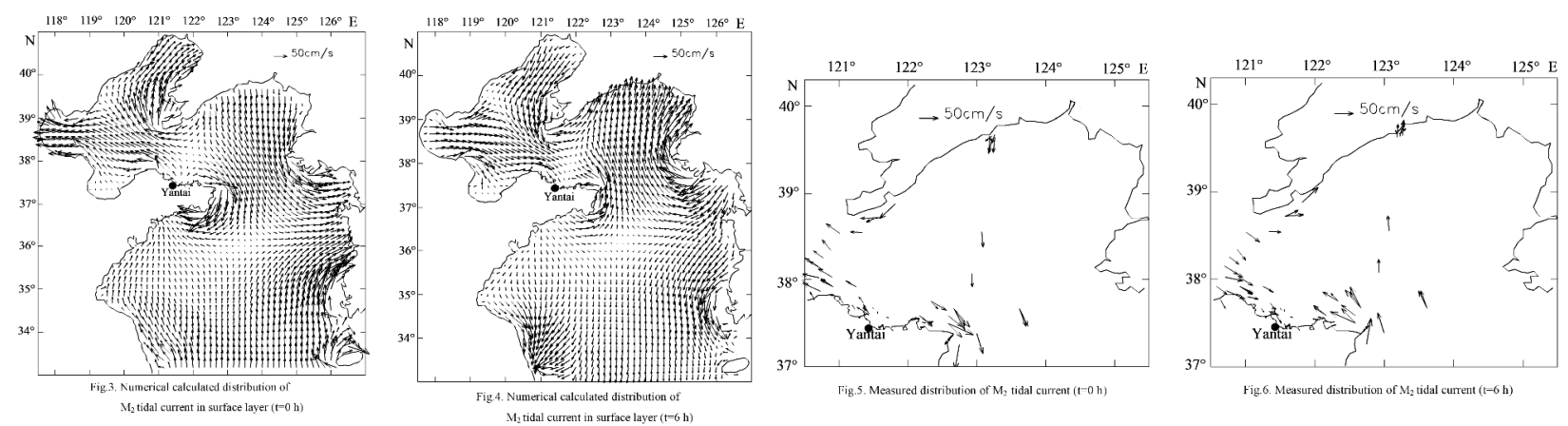

There are four $\mathrm{M}_{2}$ tide waves in the Yellow Sea and the Bohai Sea ${ }^{[8,9]}$. The computation indicates that three convergent and divergent regions of tidal currents exist at the junctures of these tidal waves.

Fig. 3 and Fig. 4 show that one of these regions is near $39.3^{\circ} \mathrm{N}, 119.1^{\circ} \mathrm{E}$ in the western part of Bohai Bay. The computations indicate that the tidal currents of $\mathrm{M}_{2}$ constituent are very weak in this sea area. 
The other region lies offshore eastwards in Yantai. Fig. 3 and Fig.5 show that tidal current of $\mathrm{M}_{2}$ constituent is divergent at $00 \mathrm{~h}$, and Fig. 4 and Fig. 6 show that it is convergent at $06 \mathrm{~h}$ in this sea area.

The third region is near $36^{\circ} \mathrm{N}, 123.3^{\circ} \mathrm{E}$ in the middle of the Yellow Sea. Fig.3 shows that divergent phenomenon appears in east-west direction, and convergent phenomenon appears in north-south direction. Fig.4 shows that convergent phenomenon appears in east-west direction, and divergent phenomenon appears in north-south direction after half period.

The calculations indicate that convergent and divergent phenomena which appear in the second layer to the fifth layer are same as in the surface layer.

The five layers tidal currents fields of $S_{2}$ constituent were computed hourly in a period based on the same method in the Yellow Sea and the Bohai Sea. The calculations indicate that convergent and divergent phenomena of the tidal currents of $\mathrm{S}_{2}$ and $\mathrm{M}_{2}$ constituents are similar.

There are two $K_{1}$ tide waves in the Yellow Sea and the Bohai Sea ${ }^{[8,9]}$, and the juncture of tidal waves is in the sea area to the east of Chengshantou. Fig.7 and Fig.8 show the numerical calculated distributions of tidal current of $\mathrm{K}_{1}$ constituent at $00 \mathrm{~h}$ and $06 \mathrm{~h}$ in surface layer. The result indicates that one convergent and divergent region of tidal currents exists at the juncture of tidal waves.
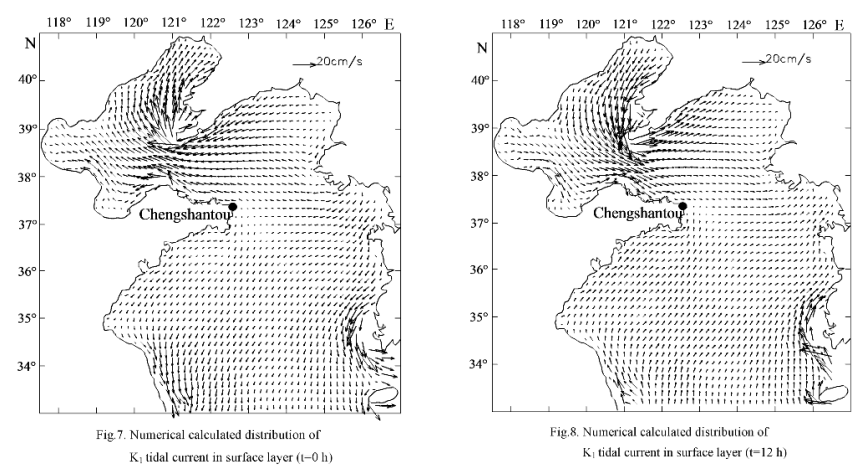

The calculations also indicate that convergent and divergent phenomena which appear in the second layer to the fifth layer are same as in the surface layer and convergent and divergent phenomena of the tidal currents of $\mathrm{O}_{1}$ and $\mathrm{K}_{1}$ constituents are similar.

\section{Effects of convergent and divergent phenomena of the tidal current constituents on tidal current types}

Fig.9 and Fig. 10 show the calculated distributions of tide and tidal current types in the Yellow Sea and the Bohai Sea.

Effects of convergent and divergent phenomena of the tidal current constituents on tidal current types are as follows:

In the convergent and divergent region of tidal currents of semidiurnal constituents near $39.3^{\circ} \mathrm{N}, 119.1^{\circ} \mathrm{E}$, tide is irregular semi-diurnal tide, and tidal current is irregular semi-diurnal tidal current. Fig.3, Fig.4, Fig.7, and Fig.8 indicate that the cause of this tidal current type lies in the fact that tidal currents of semidiurnal constituents and diurnal constituents are all weak in this sea area.

In the marginal seas adjacent to Yantai, tides and tidal currents appear to have different properties in that tides are regular semidiurnal tides, and tidal currents are diurnal currents. Therefore

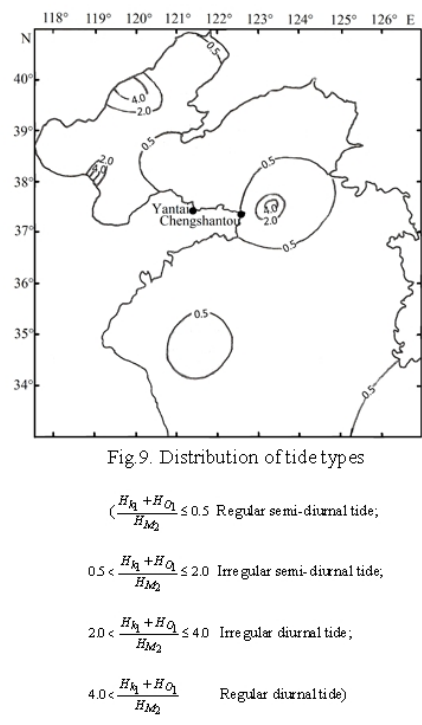

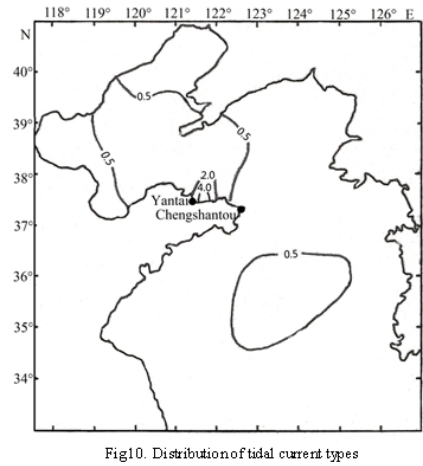

Fig10. Distributionof tidal curre rit types

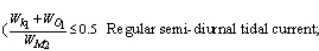

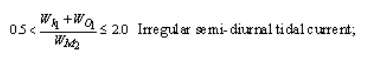

$20<\frac{W_{k_{1}}+W_{q}}{W_{M_{2}}} \leq 40$ Irreguler diurnal tidal currert;

$40<\frac{W_{1_{1}}+W_{q_{1}}}{W_{M_{2}}} \quad$ Regular diunal tidal current)

the directions of tidal currents are different in the two processes of rising tide that occur twice a day. Flood currents appear in the first process of rising tide and ebb currents appear in the next process of rising tide on the same day. This phenomenon is infrequent. The cause of this phenomenon can be explained based on Fig.3 to Fig.8. In this sea area, Fig 3 \& fig 5 show divergent currents of semidiurnal constituents, while Fig $4 \& 6$ show that 6 hours later there are periods of convergent 
currents of semidiurnal constituents. This combination of divergence and convergence makes the currents of semidiurnal constituents very weak. Conversely, in same sea area, Fig $7 \&$ Fig 8 show that there is no convergent or divergent phenomenon observed in the currents of diurnal constituents and the currents of diurnal constituents are relatively strong.

In the convergent and divergent region of tidal currents of semidiurnal constituents near $36^{\circ} \mathrm{N}$, $123.3^{\circ} \mathrm{E}$, the tide is the regular semi-diurnal tide, and the tidal current is the irregular semi-diurnal tidal current. The cause of this phenomenon can be explained by consideration of Fig.3, Fig.4, Fig.7, and Fig.8. In this sea area, the currents of semidiurnal constituents and diurnal constituents are all weak and have few differences from each other on numerical values.

In the convergent and divergent region of tidal currents of diurnal constituents to the east of Chengshantou, tides are irregular semi-diurnal tide and diurnal tide, while the tidal current is regular semi-diurnal tidal current. The causes of these phenomena are as follows: 1) an amphidromic point of semi-diurnal tide wave appears here ${ }^{[8,9]}$. The computations indicate that the tides of semidiurnal constituents are very weak, and the currents of diurnal constituents are relatively strong. 2) Fig.7 and Fig. 8 show that the currents of diurnal constituents are very weak here.

\section{Conclusions}

Three convergent and divergent regions of tidal currents exist in the junctures of four semi-diurnal tide wave systems, and one exists in the juncture of two diurnal tide wave systems. The computations indicate that the tidal current constituents are very weak here. The convergence and divergence of tidal current constituents is the probable cause of the dissimilarity between the tide type and tidal current type in these sea areas.

\section{Acknowledgments}

This paper was supported partly by the International Collaborative Research Project for outstanding university teachers of Shandong Province of China.

\section{References}

[1] Sun Y L, Chen S J and Zhao K S. A three-dimensional baroclinic model of the coastal water.I.Numerical simulation of tidal and residual currents in the Bohai Sea. Journal of Ocean University of Qingdao. 1990, 20 (3):13-24.

[2] Huang Z K. Tidal waves in the Bohai Sea and their variations. Journal of Ocean University of Qingdao. 1991,21 (2): 1-11.

[3] Dou Z X, Yang L W and Ozer J. A three-dimensional model for the simulation of tide and tidal currents in the Bohai Sea. Journal of ACTA Oceanolgica. 1993,15: 1-15.

[4] Huang D J, Su J L and Backhaus J. O. Modelling the seasonal thermal stratification and baroclinic circulation in the Bohai Sea. Journal of Continental Shelf Research.19 1999 (11):1485-1505.

[5] Li G S, Wang H L and Li BL. A model study on seasonal spatial-temporal variability of the Lagrangian Residual Circulations in the Bohai Sea. Journal of Geographical Sciences.2005,15(3): 273-285.

[6] Backhaus J O.A semi-implicit scheme for the shallow water equations for application to shelf sea modeling. Continental Shelf Research,1983,2(4):243-254

[7] Backhaus J O.A three-dimensional model for the simulation of shelf sea dynamics, Deutsche Hydrographische Zeitschrift,1985,38:165-187

[8] Fang $\mathrm{G}$ h. Tide and tidal current charts for the marginal seas adjacent to China. Journal of Oceanology and Limnology. 1986, 4(1):1-16.

[9] Editorial Board for Marine Atlas. Marine Atlas of Bohai Sea, Yellow Sea and Donghai Sea: Hydrology. China ocean press. 1992,427-433 